\title{
DNA aneuploidy in ulcerative colitis and in colorectal carcinoma - a comparative study
}

\author{
Roger Stenling ${ }^{\mathrm{a}, *}$, Bernt O. Jonsson ${ }^{\mathrm{b}}$, \\ Richard Palmqvist ${ }^{\mathrm{a}}$ and Jörgen N. Rutegård ${ }^{\mathrm{b}, \mathrm{c}}$ \\ a Department of Pathology, University Hospital of \\ Umeå, Sweden \\ ${ }^{\mathrm{b}}$ Department of Surgery, University Hospital of \\ Umeå, Sweden \\ ${ }^{\mathrm{c}}$ Department of Surgery, Örnsköldsvik Hospital, \\ Sweden
}

Received 25 July 1998

Revised 24 August 1998

Accepted 2 December 1998

DNA aneuploidy is of interest as an additive marker for carcinoma risk in ulcerative colitis. It is known that colorectal carcinomas often are aneuploid with DNA indices centered around a median value of 1.5 , corresponding to triploidy, and that adenomas, if aneuploid, have DNA indices closer to 2.0, the tetraploid region.

In a colonoscopic surveillance programme, colorectal mucosal biopsies from 104 patients with ulcerative colitis were examined by flow cytometry, and the DNA indices determined and compared with findings of cellular dysplasia. In 17 patients, DNA aneuploidy was diagnosed, with DNA indices ranging from 1.2 to 2.0, median 1.9. Three patients with high grade dysplasia all had DNA indices within the triploid region. These results were compared with the DNA indices from a group of 49 patients with non-colitis-associated aneuploid colorectal carcinomas, in which the levels ranged from 1.1 to 2.0 with a median value of 1.5 .

Accordingly, the DNA index in the colitis patients with aneuploidy was more often within the tetraploid region. These results, obtained in patients with ulcerative colitis, indicate a possible precancerous progress from diploidy over tetraploidy to triploidy also in patients with long-standing ulcerative colitis. In addition, the results speak in favour of a connection between DNA indices in the triploid region and more profound premalignant alterations.

\footnotetext{
*Corresponding author: Roger Stenling, M.D., Ph.D., Department of Pathology, Umeå University, S-901 87 Umeå, Sweden. Tel.: +46 90785 1545; Fax: +46 9078528 29; E-mail: roger.stenling.us@vll.se.
}

Keywords: Ulcerative colitis, colitis-cancer, DNA aneuploidy, flow-cytometry

\section{Introduction}

The increased risk for colorectal cancer in ulcerative colitis is well known. In surveillance programmes, high grade cellular dysplasia has emerged as the standard marker for impending or already present malignancy [15]. However, the concept of dysplasia has its shortcomings. Not all carcinomas are preceded by dysplasia, and the interpretation of the histological alterations is subjective $[9,13]$.

In the search for new markers reflecting precancerous conditions and enhanced risk for cancer development, DNA alterations determined by flow cytometry have attracted considerable interest $[13,16]$. Thus, DNA aneuploidy has been found to correlate to, and sometimes precede, dysplasia and carcinoma $[10,13$, $16,18]$.

However, DNA aneuploidy is not a uniform aberration. Instead, all quantitative DNA indices (DI) except the normally present DI 1.0 (diploidy) are regarded as DNA aneuploidy [6]. More over, a DI of 2.0 is moreover considered as the tetraploid variant of aneuploidy, although euploid polyploidisation can appear as DI 2.0 [1]. Aneuploidy is frequently observed in colorectal carcinomas, in which aneuploid DI values mainly are found in the triploid region (DI around 1.5) [3,7]. DNA content analyses within non-colitis-related adenomas and carcinomas show that near diploidy (DI around 1.0) and tetraploidy are more common in adenomas than in carcinomas, while "carcinoma in adenoma" often shows triploidy [3]. Accordingly, DNA triploidy may reflect more severe genetic alterations [17]. More recent reports associate presence of aneuploidy with loss of symmetry in cell division [4]. This is in accordance with the modern views on genetically based multistep carcinogenesis during colorectal cancer development [20,23].

The aims of the present study were to evaluate, in patients with ulcerative colitis, whether: 
1. The pattern of DI in aneuploid biopsy samples were of "adenoma or carcinoma type".

2. Findings of DI within the triploid region in aneuploid biopsy samples were related to presence of high grade dysplasia or colitis-associated cancer.

\section{Material and methods}

Within a framework of a colonscopic surveillance programme in a defined catchment area, biopsy specimens for evaluation of presence of dysplasia and DNA content were sampled from rectum and from five colonic regions at regular examinations in all patients [8]. In the earlier period of the series, some examinations were performed by sigmoidoscopy.

The specimens were analysed by DNA flow-cytometry retrospectively in patients with dysplastic or indefinite changes from 1977, and prospectively in all patients from 1984. Accordingly, the present study comprises the results from 104 patients from the time period 1977 through 1990. Specifically, we studied the distribution of DI from 63 biopsy specimens taken at 1-4 occasions from the defined colorectal regions in 17 patients with ulcerative colitis. This distribution of the DNA indices was compared with that of 75 aneuploid tumour samples from 49 patients with noncolitis-associated colorectal adenocarcinoma.

\subsection{DNA analysis}

Fresh-frozen and archival biopsy specimens were analysed with respect to DNA content. Fresh samples were immediately minced into small pieces and stored at $-80^{\circ} \mathrm{C}$ until analysis [17]. From archival samples $100 \mu \mathrm{m}$ sections were cut and deparaffinated [18]. After trypsin digestion, staining with propidium iodide and filtration [22] the nuclear suspension was run in a FACScan cytoflourograph (Becton and Dickinson, Sunnyvale, CA, USA). Evaluation of DNA histograms using DI to describe the ploidy level was performed as previously discribed $[17,18]$. Biopsies were thus classified as diploid/near diploid or aneuploid. DI of 2.0 was regarded as the tetraploid variant of aneuploidy. Internal control was represented by the non-epithelial cells (i.e., inflammatory- and stromal cells) present in each biopsy and the biopsies were denominated aneuploid when two peaks were detected and where the highest peak was above or equivalent to DI 1.1. External controls were not used in this study. In our experience, the different methods used for fresh and archival material do not influence the results. Aneuploid biopsies from the two patients analysed both retrospectively using archived material and prospectively using fresh tissue expressed equal DI, although DNA histograms from archived material showed more debris.

\subsection{Light microscopy}

Biopsy specimens were fixed in formalin, embedded in paraffin and routinely processed for light microscopy. Dysplasia was recorded as high grade (HGD), low grade (LGD), and as uncertain findings (indefinite for dysplasia, ID), according to the international standardised classification [15].

\subsection{Statistics}

Differences between groups were examined using the Mann-Whitney $U$-test. A $p$-value of less than 0.05 was required for statistical significance and two-sided tests were performed.

\section{Results}

DNA aneuploidy was diagnosed in 13 patients in the prospective surveillance, and in 6 patients in the retrospective study. Since two patients appeared in both studies, the total number of patients with aneuploidy was 17 . There were 12 men and 5 women with an age range of 23-65 years. The mean period after their inclusion in the surveillance programme was 6 years (range 1-13). Totally, 63 aneuploid biopsy specimens were found. Of the 17 patients analysed, one had presence of aneuploidy in all 6 regions of colon-rectum, two in 5 regions, three in 3 regions, one in two regions, and the remaning 10 patients in one region. Of the 104 patients with ulcerative colitis totally included in the study dysplasia was recorded in 31. ID was found in 7 patients, LGD in 19 and HGD in 5.

Dysplasia (LGD or HGD) or indefinite changes (ID) were diagnosed in $\mathbf{x x x}$ of the 104 patients included in the study. Of the 17 patients with ulcerative colitis and DNA aneuploidy, 12 showed presence of dysplasia. HGD was found in 6 biopsies from $3 / 12$ patients while LGD and/or ID was observed in 15 biopsies from 9/12 patients (Fig. 1). The DI observed in the patients with HGD were all within the triploid region (DI 1.41.6). In addition to the patients with HGD, 5 more patients had DI within the triploid region, one with LGD or ID, and 4 without any sign of dysplasia. 


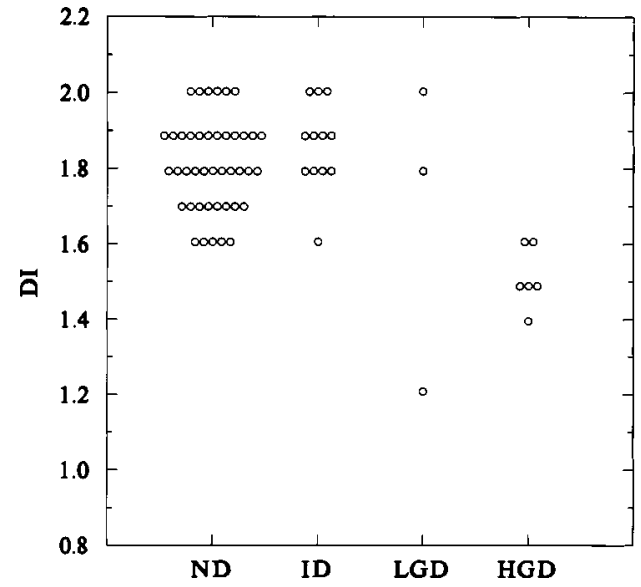

Fig. 1. DNA index (DI) in 63 aneuploid biopsy samples from 17 patients with long-standing ulcerative colitis. The distribution of DI, presented as mean values of each aneuploid sampling region per patient, is related to presence of high grad dysplasia (HGD), low grade dysplasia (LGD), indefinite for dysplasia (ID) and to no dysplastic findings (ND).

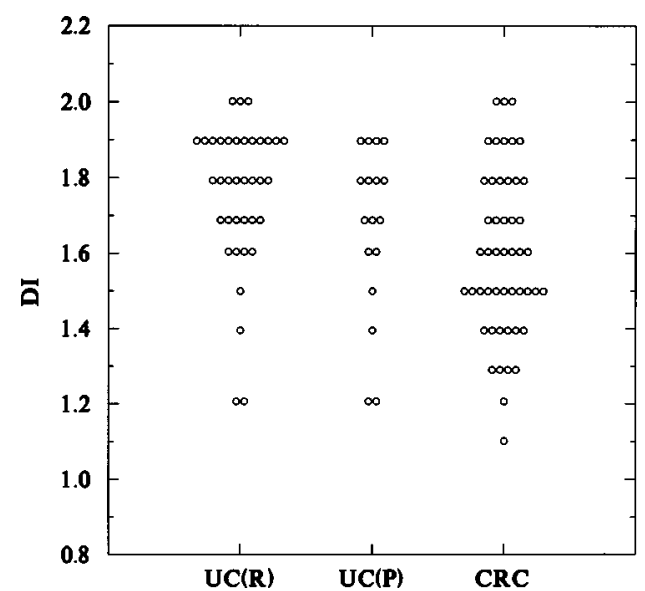

Fig. 2. Comparison of DNA index (DI) in 63 aneuploid biopsy samples from 17 patients with long standing ulcerative colitis (UC) to 75 aneuploid tissue samples from 49 patients with non-colitis colorectal carcinoma (CRC). The distribution of aneuploid DI in UC is presented as mean values of each aneuploid colorectal region per patient, UC (R). In CRC, the distribution is presented as mean values from each carcinoma. As a completion, the mean values of aneuploid samples from each patient is also illustrated, UC (P).

One patient with HGD and DI 1.5 refused proposed surgical treatment. He developed two carcinomas found at autopsy two years later. The time interval between biopsy and autopsy was two years.

For aneuploid samples in patients with ulcerative colitis the DI were distributed between 1.2 and 2.0 with a mean value of $1.8 \pm 0.19$, when expressed as mean values of each aneuploid region per patient. For the aneuploid non-colitis-associated carcinomas, the DI were distributed between 1.1 and 2.0 with a mean value of $1.6 \pm 0.22$.

When comparing the distributions of abnormal DI values from the patients with ulcerative colitis with the frequency of DI values from the patients with noncolitis-associated carcinoma, the former was significantly $(p<0.001)$ more commonly localised within the tetraploid region, in contrast to the latter, in which DI values within the triploid region dominated (Fig. 2).

\section{Discussion}

The DI values from patients with ulcerative colitis were more commonly localised within the tetraploid region [4,21]. In patients with the non-colitis associated carcinomas DI values from the triploid region dominated. Accordingly, the pattern of DI in aneuploid biopsies from patients with ulcerative colitis were predominantly of "adenoma-type". However, findings of DI within the triploid region in colitis patients appeared related to presence of cancer as well as high grade dysplasia. This supports the view of neoplastic progression of DNA content to the triploid interval, as has been postulated for adenomas [7], and which has earlier been indicated by Rubin et al. [16] in an investigation of patients with ulcerative colitis. Whether such a process is initiated by endoreduplication of cells with slightly hypoploid DNA content and with subsequent loss of genetic material $[16,19]$ or corresponds to later views with loss of symmetry in cell division [4] correlated to multistep carcinogenesis [20] with reported genetic alterations [23] can not be interpreted from the results of this study. It might also be noted that euploid polyploidisation can occur in regenerative epithelium and therefore, some of our tetraploid observations might be explained by such a mechanism [1].

In the search for markers of cancer risk, more discriminating than dysplasia, in patients with ulcerative colitis, diagnosis of DNA aneuploidy has been deemed as an important step forward $[5,10,14,16]$. The results from the present series, with an accumulation of triploid DI values in patients with HGD, indicate a connection between DI in the triploid region and presence of more profound morphologic premalignant alterations. Accordingly, a possibility to further increase the cancer predictive value of findings of findings of an abnormal DNA content might exit. 


\section{Acknowledgements}

This study was supported by grants from the Research Foundation,Umeå University, the Swedish Cancer Society, Project No 2520-B96-10XCC, and the Swedish Society of Medicine, Project No 665/90.

\section{References}

[1] S. Biesterfeld, L. Füzesi, F. Härle et al., DNA-cytometric detection of euploid polyploidisation in oral lichen ruber planus, Analyt. Quant. Cytol. 13 (1991), 7-10.

[2] J.B. Fozard, P. Quirke, M.F. Dixon, G.R. Giles and C.C. Bird, DNA aneuploidy in ulcerative colitis, Gut 27 (1986), 14141418 .

[3] W. Giaretti, S. Sciallero, S. Bruno, E. Geido, H. Aste and A. Di Vinci, DNA flow cytometry of endoscopically examined colorectal adenomas and adenocarcinomas, Cytometry 9 (1988), 238-244.

[4] W. Giaretti, A model of DNA aneuploidization and evolution in colorectal cancer, Lab. Invest. 71 (1994), 904-910.

[5] C. Hammarberg, P. Slezak and B. Tribukait, Early detection of malignancy in ulcerative colitis. A flow-cytometric study, Cancer 53 (1984), 291-295.

[6] W. Hiddemann, J. Schumann, M. Andreef et al., Convention on nomenclature for DNA cytometry, Cancer Genet. Cytogenet. 13 (1984), 181-183.

[7] W. Hiddemann, D. von Bassewitz, H.-J. Kleinemeier et al., DNA stemline heterogeneity in colorectal cancer, Cancer $\mathbf{5 8}$ (1986), 258-263.

[8] B. Jonsson, L. Åhsgren, L.O. Andersson et al., Colorectal cancer surveillance in patients with ulcerative colitis, $B r$. J. Surgery 81 (1994), 689-691.

[9] J.E. Lennard-Jones, B.C. Morson, J.K. Ritchie and C.B. Williams, Cancer surveillance in ulcerative colitis. Experience over 15 years, Lancet II (1983), 149-152.

[10] R. Löfberg, O. Broström, P. Karlén, Å. Öst and B. Tribukait, DNA aneuploidy in ulcerative colitis: reproducibility, topographic distribution, and relation to dysplasia, Gastroenterology 102 (1992), 1149-1154.

[11] J.B.D. McHugh, J.D. Bozdech, G.H. Warren, C.R.D. Meyer and D.J. Ahnen, Aneuploidy in a large cohort of patients with chronic ulcerative colitis in ongoing surveillance programs, Gastroenterology 100 (1991), A230.
[12] G.I. Meling, O.P.F. Calausen, A. Bergan, A.A. Schjölberg and T.O. Rognum, Flow cytometric DNA ploidy pattern in dysplastic mucosa, and in primary and metastatic carcinomas in patients with longstanding ulcerative colitis, Br. J. Cancer 64 (1991), 339-344.

[13] D.M. Melville, J.R. Jass, N.A. Shepherd et al., Dysplasia and deoxyribonucleic acid aneuploidy in the assessment of precancerous alterations in chronic ulcerative colitis. Observer variation and correlations, Gastroenterology 95 (1988), 668-675.

[14] R. Porschen, G. Molsberger, C. Reis et al., DNA - Ploidie und Dysplasie bei Colitis ulcerosa-Interimsanalyse einer prospektiven Studie, Z. Gastroenterol. 30 (1992), 857-862.

[15] R.H. Riddell, H. Goldman, D.F. Ransohoff et al., Dysplasia in inflammatory bowel disease: standardized classification with provisional clinical applications, Human Pathology 14 (1983), 931-968.

[16] C. Rubin, R.C. Haggitt, G.C. Burmer et al., DNA aneuploidy in colonic biopsies predicts future development of dysplasia in ulcerative colitis, Gastroenterology 103 (1992), 1611-1620.

[17] J. Rutegård, L. Åhsgren, R. Stenling and G. Roos, DNA content in ulcerative colitis. Flow cytometric analysis in a patient series from a defined area, Dis. Colon Rectum (1988), 710-715.

[18] J. Rutegård, L. Åhsgren, R. Stenling and G. Roos, DNA content and mucosal dysplasia in ulcerative colitis. Flow cytometric analysis in patients with dysplastic or indefinite morphologic changes in the colorectal mucosa, Dis. Colon Rectum 32 (1989), 1055-1059.

[19] S.E. Shackney, C.A. Smith, B.W. Miller et al., Model for the genetic evolution of human solid tumors, Cancer Research 49 (1989), 3344-3354.

[20] T. Sugimura, Multistep carcinogenesis. A 1992 perspective, Science 258 (1992), 603-607.

[21] B. Tribukait, C. Hammarberg and C. Rubio, Ploidy and proliferation patterns in colorectal adenocarcinomas related to Dukes'classification and to histopathological differentiation, Acta Path. Microbiol. Scand. Sect. A 91 (1983), 89-95.

[22] L.L. Vindelöv, I.J. Christensen and N.I. Nissen, A detergent trypsin method for the preparation of nuclei for flow cytometric DNA analysis, Cytometry 3 (1983), 323-327.

[23] B. Vogelstein, E.R. Fearon, S.R. Hamilton et al., Genetic alterations during colorectal-tumor development, New. Engl. J. Med. 319 (1988), 525-532. 


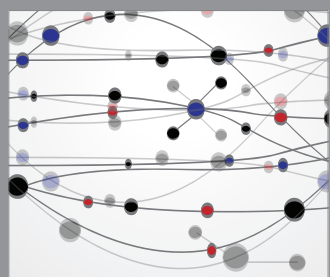

The Scientific World Journal
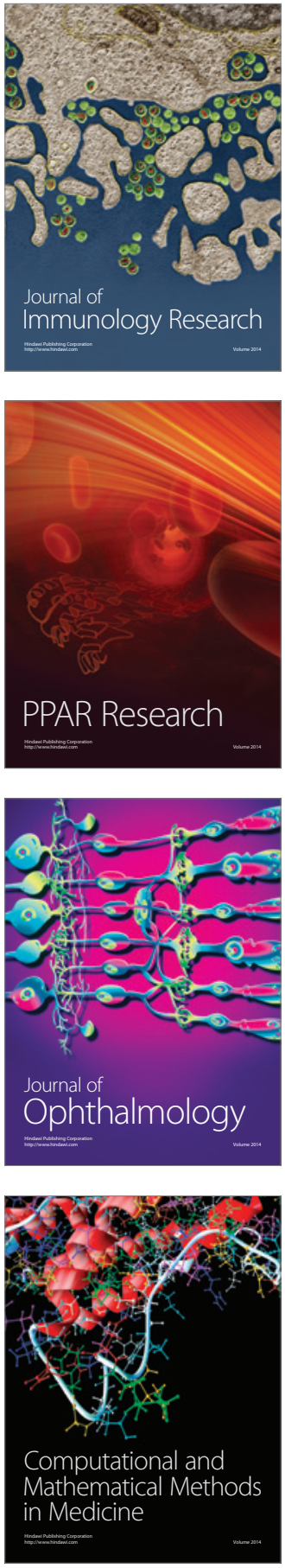

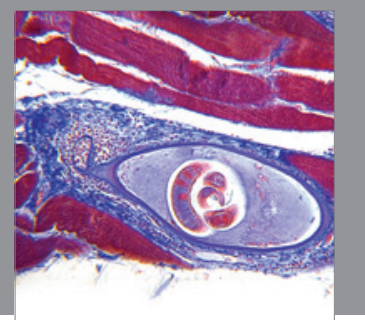

Gastroenterology

Research and Practice
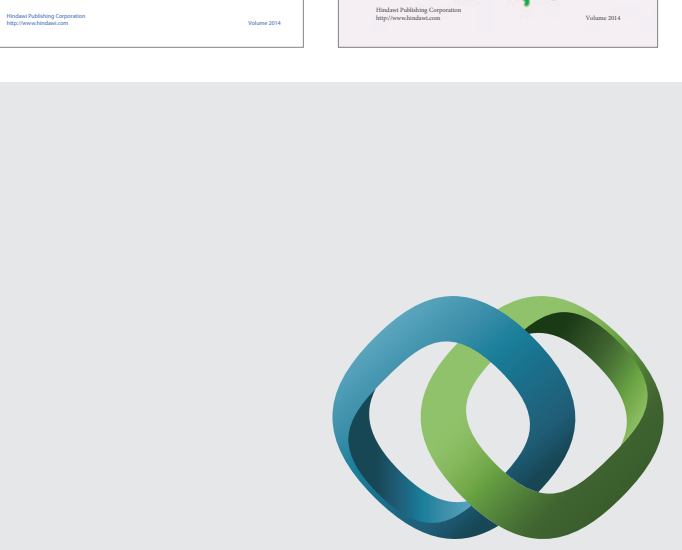

\section{Hindawi}

Submit your manuscripts at

http://www.hindawi.com
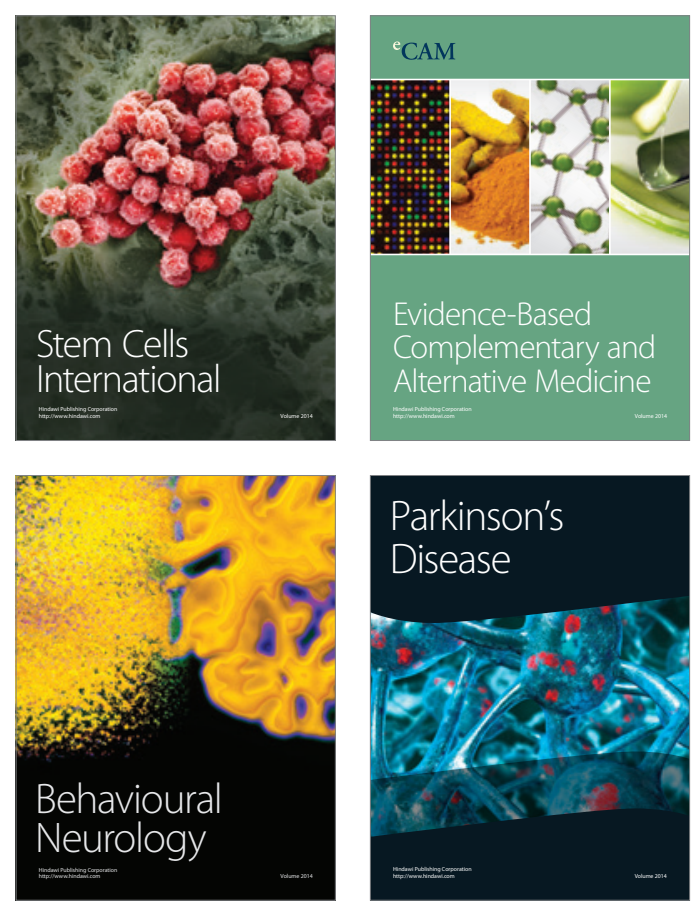

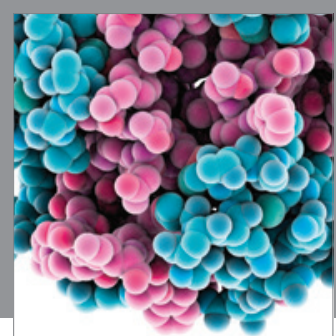

Journal of
Diabetes Research

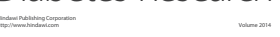

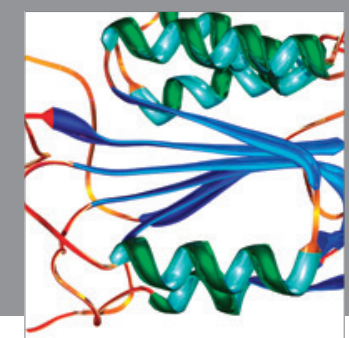

Disease Markers
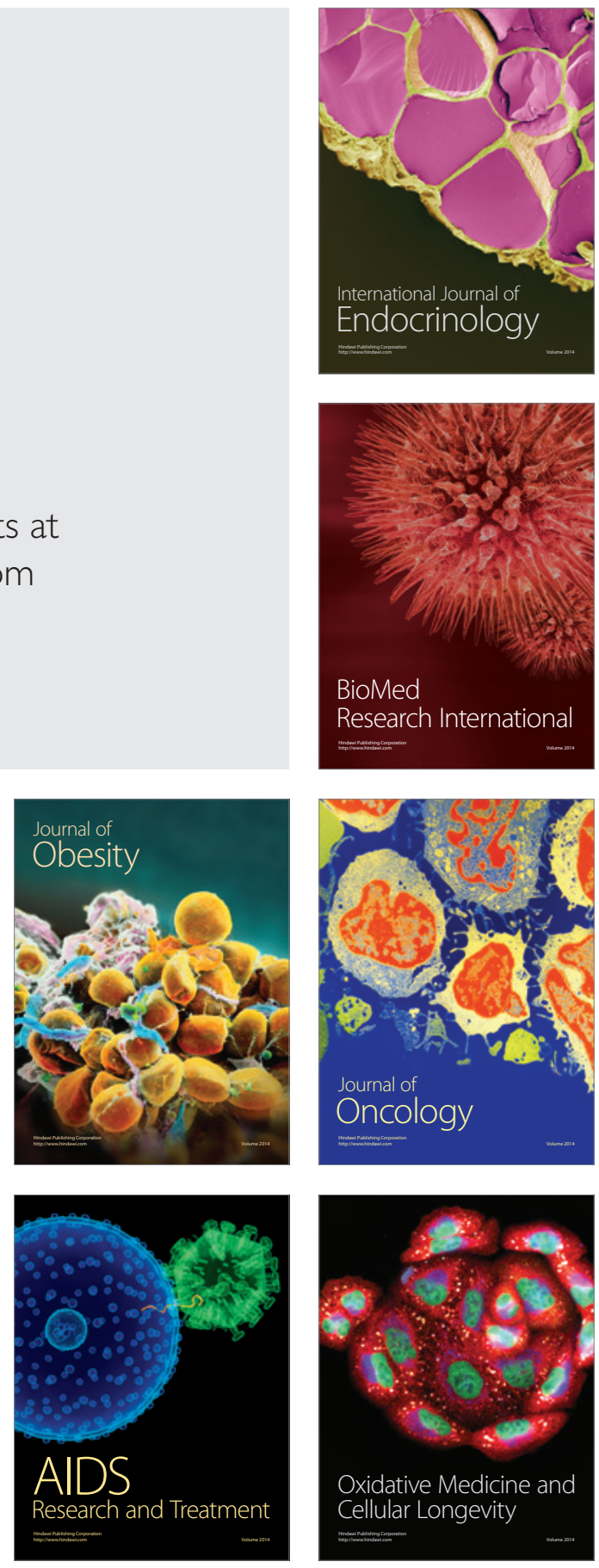